\title{
STOCHASTIC MODEL FOR ELECTRIC FIELD-INDUCED MEMBRANE PORES
}

\section{ELECTROPORATION}

\author{
I.P. SUGAR and E. NEUMANN * \\ Max-Planck-Institut für Biochemie. D-8033 Martinsried/München. F.R.G.. und Institure of Biophysics, Semmelweis Medical \\ Unicersity. Budapest 1444, P.O. Box 263. Hungary.
}

Received 16th September 1983

Accepted 8th December 1983

Key uords: Lipid bilayer: Membrane pore: Electric field: Electroporation

\begin{abstract}
Electric impulses (1-20 kV cm-1, $1-5 \mu \mathrm{s})$ cause transient structural changes in biological membranes and lipid bilayers. leading to apparentiy reversible pore formation (electroporation) with cross-membrane material flow and, if two membranes are in contact. to irreversible membrane fusion (electrofusion). The fundamental process oferative in electroporation and electrofusion is treated in terms of a periodic lipid block model. a block being a nearest-neighbour pair of lipid molecules in either of two states: (i) the polar head group in the bilayer plane or (ii) facing the centre of a pore (or defect site). The number of blocks in the pore wall is the stochastic variable of the mociel describing pore size and stability. The Helmholtz free energy function characterizing the transition probabilities of the various pore states contains the surface energies of the pore wall and the planar bilayer and. if an electric field is present, also a dielectric polarization term (dominated by the polarization of the water layer adjacent to the pore wall). Assuining a Poisson process the average number of blocks in a pore wall is given by the solution of a non-linear differential equation. At subcritical electric fields the average pore size is stationary and very small. At supercritical field strengths the pore radius increases and, reaching a critical pore size, the membrane tuptures (dielectric breakdown). If, however, the electric field is switched off, before the critical pore radius is reached. the pore apparently completely reseals to the closed bilayer configuration (reversible electroporation).
\end{abstract}

\section{Introduction}

Biological membranes are known to become transiently more permeable by the action of short electric field pulses $[1,2]$ when a certain threshold value of the field strength is exceeded [1]. The electrically induced permeability increase leads to a transient exchange of matter across the perturbed nembrane structures. When two membranes are in close contact with each other electric fields not only enhance material exchange but also cause membrane-membrane fusion [3-5]. A further aspect of electric field effects on membrane structures is the artificial transfer of macromole-

- To whom correspondence should be addressed at: Physical and Biophysical Chemistry, University of Biclefeld, P.O. Box 8640, D-4800 Bielefeld 1, F.R.G. cules or particles into the interior of biological cells and organelles [6,7]. Recently, it was shown that homogeneous electric fields can be used to transfect culture cells in suspension with foreign genes [8].

The mechanism of electrically induced membrane permeability changes is not known. There are various proposals and estimates $[1,6-12]$. It appears that high electric fields cause pores to form in the membranes [1-14]; the fundamentals of a general thermodynamic treatment of electric pore formation (electroporation) were given [8].

In any case, the primary action of electric fields is on the charges and dipoles of the membrane, favouring charge and dipole configurations associated with a larger overall dipole moment. This in turn may lead to a thinning of membrane patches 
and to an increase in the defects in the membrane structure and. finally, to the formation of holes [8.11].

Molecularly, in the framework of the inverted pore model [11]. the pore edge is assumed to be a curved half-toroid surface [11.14-16]. This edge concept [15] may have been borrowed from the Hartley model for micelles [17]. In particular. Fromherz [18] has stressed that the Hartley model is not consistent with the experimental data. Here we present a new model for a bilayer pore structure: the periodic block model. According to this model, the pore wall contains blocks of two lipids which are rotated $90^{\circ}$ compared to the lipid position in normal planar bilayers.

The periodic block model is shown to describe quantitatively the essential features of electric pore formation in planar and vesicular lipid bilayer membranes.

\section{The block model for a pore}

A pore in a lipid bilayer may be viewed to consist of a pore wall and the pore interior. In our block model the pore wall is a periodic arrangement of lipids in normal bilayer position and of rotated lipid blocks (fig. 1). A block is defined by two nearest-neighbour lipids within a layer of the bilayer. A rotated block contains two lipids tilted hy about $90^{\circ}$ relative to the normal lipid position in a layer. Generally, the size of a block may depend on the number. $p$. of lipid molecules necessary to just cover the hydrophobic part of another lipid that is perpendicular to the former ones (fig. 2 ). Thus $p=h / l$ where $h$ is the length of the hydroplobic part and / the average 'thickness' of the lipid. For eholesterol $h=1.65 \mathrm{~nm}$ and $l=0.9$ nan [19]. hence $p \approx 2$.

Although the block rotation (fig. 2) brings the hyelration water of the polar head group into the membrane interior. this energetically unfavourable configuration becomes stable in the presence of a transmembrane voltage.

In the periodic block structure the periodic seyuence of a $90^{\circ}$-rotalcd block and a row of lipids in normal bilayer position is energetically favoured compared to an aperiodic sequence. The (a)

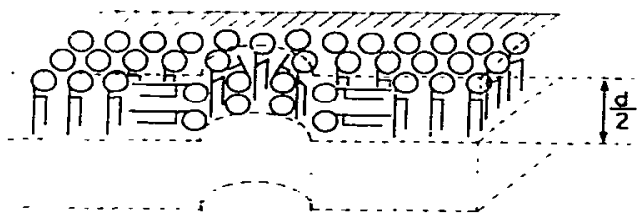

(b)

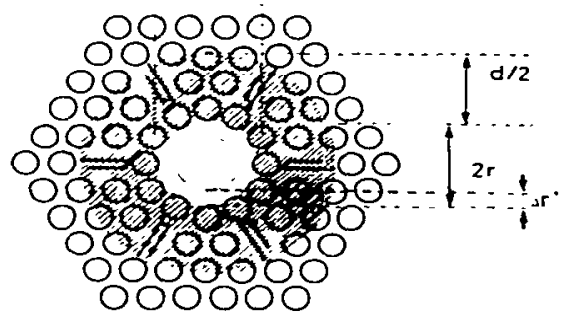

Fig. I. Periodic block structure of a section (upper layer) of a pore in a lipid bilayer. (a) Cross-section; the circles represent the polar head groups of the membrane lipids. (b) Top view of the pore mouth; the shaded area represents the planar part of the pore vall: $d$ is the thickness of the bilayer. $r$ the ratdius of the pore and $J r^{*}$ the thickness of the witter layer adjacent to the wall edge.

periodic structure ensures that the apolar parts of the lipid molecules which are in the nembrane/ water interface are everywhere surrounded by the polar head groups of the neighbours. This head group envionment reduces the extent of direct exposure of hydrophobic groups to water. Although the ordered structure of the water in the membrane/water interface is loosened it is certainly not completely interrupted. If two neighbours in the inner surface of the wall (edge) are two adjacent blocks of rotated lipids or two lipids in normal bilayer position, in both cases apolar groups are exposed to solvent to a larger extent. For the sake of a rough comparison the periodic block structure of the wall may be associated with the surface tension $\left(\gamma_{\mathrm{m}}\right)$ of the cholesterol bilayer/water $\left(\gamma_{\mathrm{m}}=2 \times 10^{-3} \mathrm{~N} / \mathrm{m}[11,19]\right)$. The surface tension characteristic for the aperiodic block structure may be clr.ser to the value $\gamma_{10 / 1} \approx$ $0.05 \mathrm{~N} / \mathrm{m}$ of the oil/wat.; interface [19]. Since $\gamma_{11} \ll \gamma_{\text {o, }}$, the periodic block structure appears to be favoured. 
(a)

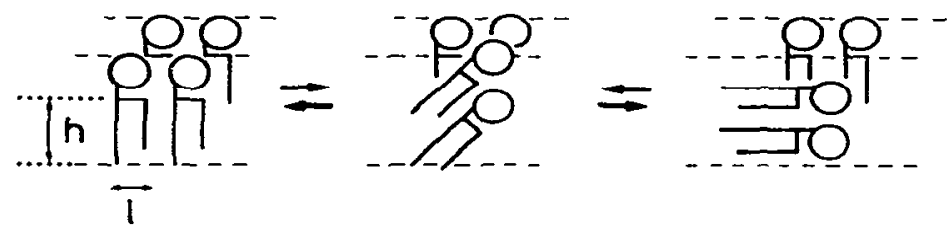

(b)

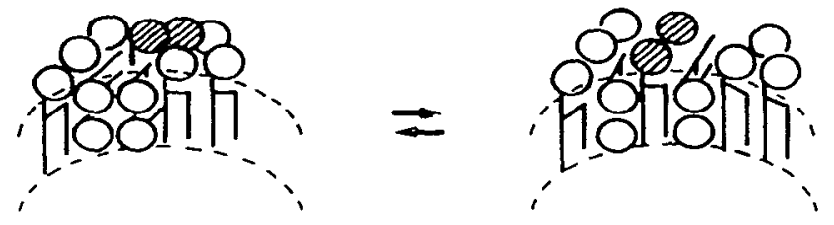

(c)
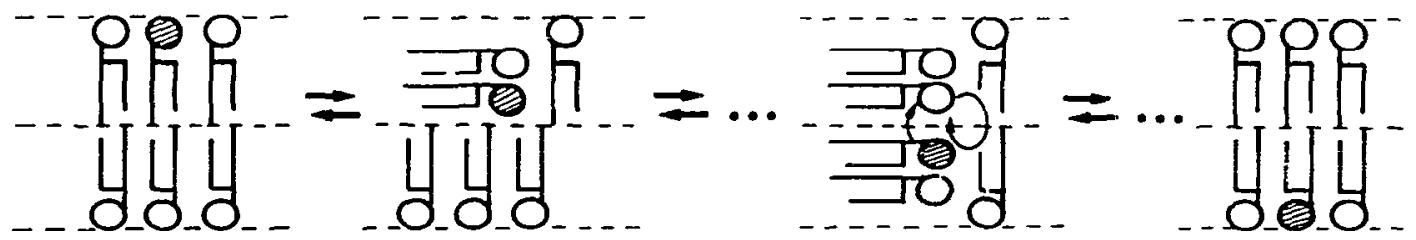

Fig. 2. Elemental 'block transitions' in a lipid layer of a planar bilayer membrane (a) Rotatic:a of a blo-k (of two lipids) by approx. $90^{\circ}$ with one (possible) intermediate state: $t h$ is the length of the hydrophobic part of the lipid molecule ant / the thickness of the lipid molecule. (b) Shift of lipids from bulk layer into the pore wall (lateral diffusion) to (stepwise) form ..n additional block in the wall, The two shifting lipids considered here are shaded. (c) Block model for the flip.flop mechanism in lipid bilayers.

\subsection{Pore size}

The wall of a pore in the bilayer may be viewed as a hollow cylinder. The surfaces of this cylinder. which are exposed to water, are two rings of inner radius $r$ and outer radius $r+d / 2$. where $d$ is the bilayer thickness, and the inner surface of the pore (edge surface") of the area $2 \pi r d$. The inner circumference of the cylindrical pore is $2 \pi r$. It is useful to define the pore size $N$ by

$N=2 \pi r / l$

where $l$ is the 'average thickness' of a lipid molecule in contact with other ones. Thus, the pore size $N$ is the number of 'blocks' and lipids in normal bilayer position along the contour line of the circumference $2 \pi r$. Note that the total number of blocks in a pore wall is $2 \mathrm{~N}$ : the pore model in fig. 1 is characterized by the pore size $N=12$ with 24 blocks.

For comparison with the so-called edge energy of other pore models [11,14-16j. it is noted trat the surface tension of our poie model. $\gamma_{p}$. refers to both the inner pore surface $2 \pi r d$ as well as to the two planar ring suifaces $\pi\left[(r+d / 2)^{2}-r^{2}\right]$ (fig. 1 ) because in both regions apoiar molecular parts are exposed to solvent.

\subsection{Elemental block transitions}

The block rotation by approx. $90^{\circ}$ represented in fig. 2a appears a sterically possible, stepwise process because the length $(h)$ and width $(2 l)$ of the block are comparable. The course of the rota- 
tion may include transient changes in the relative position (shearing) of the hydrophobic lipid tails of the two lipids of the block. At $90^{\circ}$ there is maximum contact between a rotated block and the neighbouring lipids in normal bilayer position.

The biock shift shown in fig. $2 b$ is the lateral diffusion of two lipids into the pore edge such that a new block increases the pore size from $N$ io $N+1$.

\section{3. $r \% p-f l o p$}

Our block model and the two types of lipid movements. block rotation and shift. may also be used as a hasis for a new interpretation of the flip-flop phenomerion of (slow) lipid exchange between the two layers of a bilaver. The sequence of events comprising a flip-flop may be viewed as in fig. 2c. including two block rotations and lipid exchange in the plane of the edge area of a pore or of another "defect site" in a bilayer. In the proposed mechanism mip-flop would also be enhanced in the presence of a transmembrane voltage.

\subsection{Siate aransitioms in a pore}

The periodic block structure may be described in terms of pore states $a(a=0,1,2,3 . \ldots)$ related whe pore size $N$ by $2 a=N$. In fig. 3 the enlargement of a pore is described in terms of a state transition from $a$ to $a+1$. In our block model of pore formation this transition occurs through two energetically unfavourable intermediates. At first a 'biock shift' increases the pore size $N$ by 1 ; the free energy barrier for this step being $\alpha$. representing two adjacent blocks in the same 'direction'. (The barrier for an initial block rotation within the periodic block structure $(N, a)$ is larger than $\alpha$ because in this case three blocks would be in the same direction within the pore wall.) The block shift $(N \rightarrow N+1)$ is followed by a block rotation in the pore wall. The rotation changes neither the pore size nor the (unfavourable) free energy level (see fig. 3). A second shift of lipids in between the two rotated block builds up again an energetically favourable periodic state $(a+1)$ with $(N+2)$ blocks. If $\alpha \gg k T$ (the molecular thermal energy). pore formation is a rare event. The extremely low conductivity of planar bilayers at low transmembrane voltage confirms the inequality $\alpha \gg k T$.

\section{Thermodynamics of electroporation in planar bilayers}

The conductivity changes in planar lipid bilayers. observed when the transmembrane voltage exceeds a threshold value, have been analyzed in terms of membrane pores [11.20]. In an attempt to describe thermodynamically the electric field-induced pore formation (electroporation) let us recall the Mueller-Rudin bilayer system (fig- 4 ). Here.

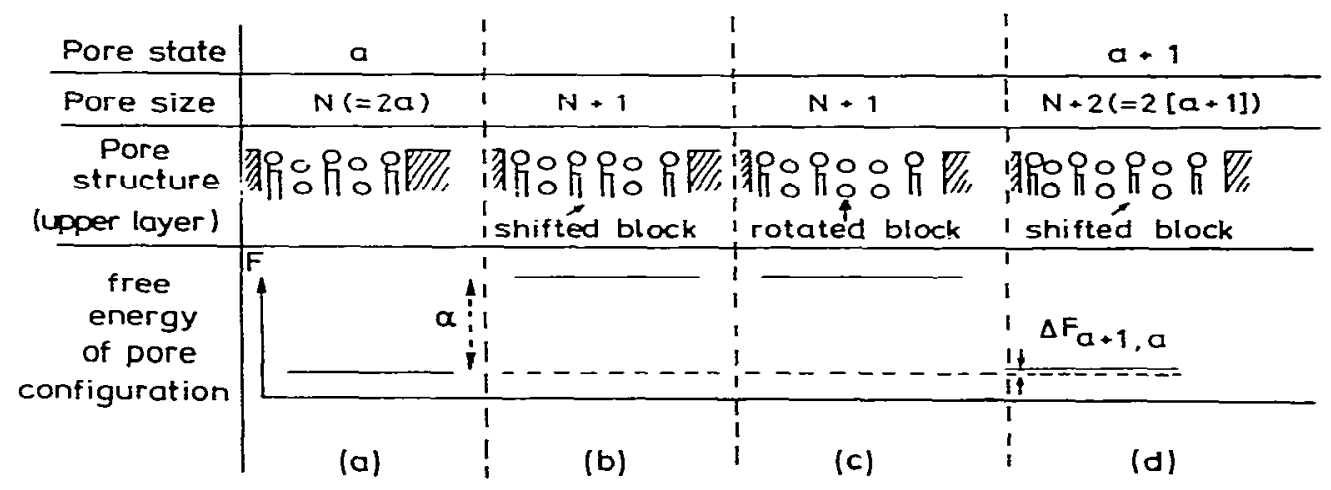

Fig. 3. Henent:art steps during pore formation in the upper layer of the bilayer membrane. Helmholtz free energy changes associated with the intermediate steps betheen two periodic pore states. 
(a)

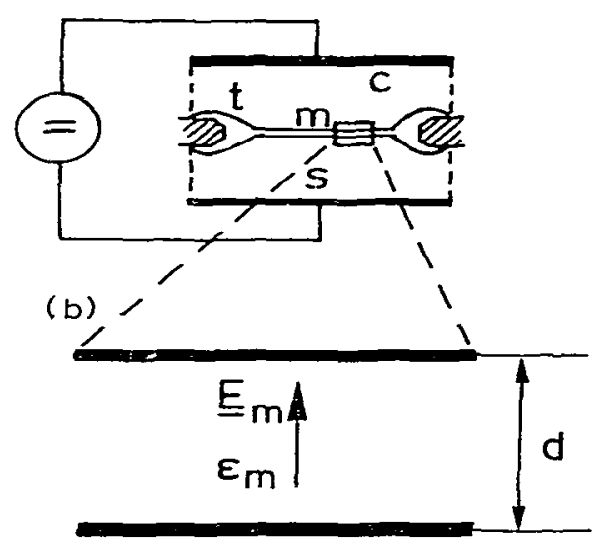

(c)

(d)

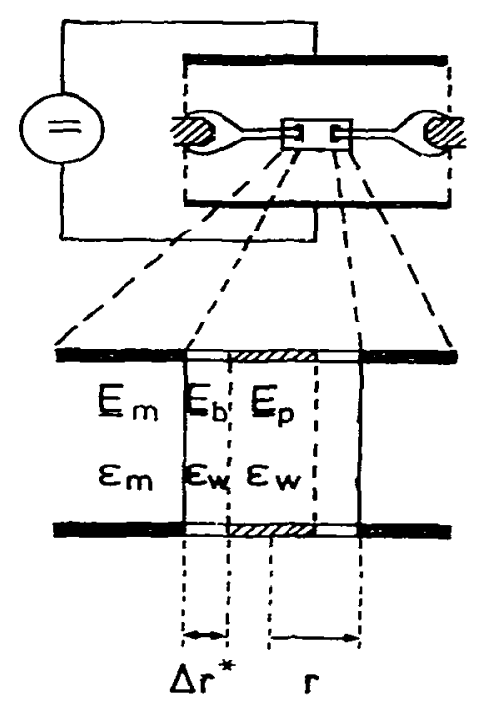

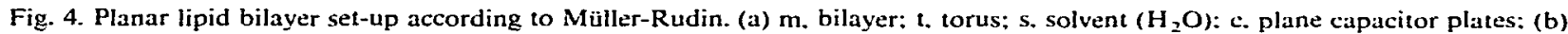
enlarged section of an intact bilayer (without pores): $E_{\mathrm{m}}$. electric field strength in the bilayer caused by the applied voltage of the capacitor; $\epsilon_{\mathrm{m}}$. relative dielectric permittivity of the bilayer; $d$, thickness of the bilayer. (c) Bilayer with a single pore: (d) enlarged section of the pore of radius $r ; E_{\mathrm{b}}$. electric field strength in the border range of thickness $\Delta r^{*}$ adjacent to the pore wall: $E_{\mathrm{r}}$. electric field strength in the inner part of the pore; $\epsilon_{n}$. relative dielectric permittivity of the solvent $\left(\mathrm{H}_{2} \mathrm{O}\right)$.

the electroporation process occurs in a closed two-phase system: the planar lipid bilayer and the torus on the one hand and the aqueous electrolyte solution bathing the lipid phase in between a planar plate condensor on the other.

Since during electroporation volume changes may occur (electrostriction) the appropriate thermodynamic work function is the Helmholtz free energy $F$. In a homogeneous electric field the change in the Helmholtz function is given by [21.22]:

$\mathrm{d} F=-5 \mathrm{~d} T-P \mathrm{~d} v+\sum \mu, \mathrm{d} n_{j}+\gamma \mathrm{d} A+E \mathrm{~d} M$

where $S$ is the entropy, $T$ the absolute temperature, $P$ the pressure, $v$ the volume, and $\mu_{j}$ and $n_{j}$ the chemical potential and the amount (in mol) of component $j$ of the homogeneous phase, respectively; $\gamma$ is the surface tension and $A$ the surface area; $M$ is the overall electric dipole moment (parallel to $E$ in plate condensor geometry; fig. 4).

In order to have the electric field strength as an independent variable, a transformation of $F$ to the (for electric field effects) characteristic Helmholtz free energy $\tilde{F}$ is necessary $[21,22]$ according to

$\mathrm{d} \bar{F}=\mathrm{d} F-\mathrm{d}(E M)$

Noting that $\mathrm{d}(E M)=E \mathrm{~d} M+M \mathrm{~d} E$ suistitution of eq. 2 into 3 yields

$\mathrm{d} F=-S \mathrm{~d} T-P \mathrm{~d} v+\sum \mu, \mathrm{d} n,+\gamma \mathrm{d} A-M \mathrm{~d} E$

It is recalled that it is $\bar{F}$ (and not $F$ ) that has a minimum at equilibrium in the presence of an externally applied electric field [22]. For a multiphase system the total change in $\bar{F}$ is given by

$\mathrm{d} F=-S \mathrm{~d} T-P \mathrm{~d} v+\sum_{\alpha} \sum_{j} \mu_{j}^{\alpha} \mathrm{d} n_{j}^{\alpha}+\sum_{J} \gamma^{\sigma} \mathrm{d} A^{\sigma}-\sum_{\alpha} M^{\alpha} \mathrm{d} E^{\alpha}$

where the summations are over all phases $\alpha$ and interfaces $\sigma$.

In the simplest case the electroporation process is considered at a constant voltage $i$ between the 
condensor plates. at constant temperature and constant external pressure. During pore formation lipid molecules move from the metastable bilayer phase [11] of volume $t_{m}$ into the torus of volume $v_{1}$ - At the same time solvent molecules 'fill' the pore voiume $v_{\mathrm{p}}$ from the bulk solvent phase of volume $v_{1}$. Because $v_{\mathrm{t}} \gg v_{\mathrm{m}}$ and $v_{s} \gg v_{p}$ both the torus and the bulk solvent phase may be considered as large reservoirs. Therefore, the chemical potentials $\mu_{1}$ and $\mu_{3}$ of the lipid and the solvent molecules, respectively, follow the approximations $\mu_{t, m}=\mu_{1.1}$ and $\mu_{4, p}=\mu_{s^{-}}$. During pore formation the system remains closed. hence $\mathrm{d} n_{\mathrm{t} . \mathrm{t}}=-\mathrm{d} n_{1, \mathrm{~m}}$ and $d n_{-p}=-\mathrm{d} n_{*}$. Therefore. in eq. 5 we may approximate $P \mathrm{~d} v=0$ and $\sum_{\mathrm{d}} \sum_{,} \mu_{1}^{\mathrm{tx}} \mathrm{d} n_{j}^{\mathrm{\alpha}}=0$. Under these conditions and at consiant temperature. pressure and volume. eq. 5 reduces to

$(\mathrm{d} F)_{r_{*}}=\sum_{n} \gamma^{n} \mathrm{~d} A^{n}-\sum_{n} A^{\prime \prime} \mathrm{d} E^{*}$

For a single pore (p) the change in the characterisiic Heimholtz free energy may be written in two termis:

\lrcorner$\left.\tilde{F}(p)=\lrcorner \tilde{F}_{\mathrm{r}}+\right\lrcorner \bar{F}_{\mathrm{cl}}$

where for the two-phase system the surface tension term is given by

\lrcorner$\left.\dot{F}_{r}=\gamma_{p}+A_{r}+\gamma_{m}\right\lrcorner \cdot A_{m}$

and the electric polarization term is expressed as

$J E_{\mathrm{e} i}=-\int M_{\mathrm{r}} \mathrm{d} E_{\mathrm{r}}-\int M_{\mathrm{m}} \mathrm{d} E_{\mathrm{m}}$

The subscripts $p$ and $m$ refer to the pore region ( $p$ ) and the normal planar bilayer $(m)$. respectively.

\section{i. I. Interfacial tension terms}

In our pore model (fig. 1) the pore surface $\Delta A_{\mathrm{p}}$ associated with $\gamma_{p}$ has two contributions. In addition to the cylindrical inner surface $2 \pi \cdot r \cdot d$ there are the two rings of the planar part of the pore wall. Hence

$d t_{r}=2 \pi r d+2 \pi\left[(r+d / 2)^{2}-r^{2}\right]$

During pore formation the surface $\Delta A_{\mathrm{m}}$ of the two circle areas $\pi(r+d / 2)^{2}$ disappears from the bi-
Iayer region associated with $\gamma_{m}$. Thus

$\Delta A_{m}=-2 \pi(r+d / 2)^{2}$

The relative change in the torus surface may be assumed to be negligibly small in view of $v_{1} \gg v_{\mathrm{m}}$; the large torus volume increases by the small value of $v_{\mathrm{p}}$.

We now introduce the pore size $N$ from eq. 1 into eqs. 10 and 11 and substitute into eq. 8 . After term rearrangement.

$\therefore \bar{F}_{\gamma}=\beta_{0} N^{2}+x_{0} N+C_{0}$

where the definitions

$\beta_{0}=-\gamma_{m} l^{2} /(2 \pi)$

$x_{1}=\left(2 \gamma_{\mathrm{p}}-\gamma_{m}\right) / d$

$C_{0}=\left(\gamma_{p}-\gamma_{m}\right) \pi d^{2} / 2$

are utilized. The extrapolation to zero pore size. i.e., $r=0 . \quad N=0$, formally yields $\Delta \tilde{F}_{\gamma}=\left(\gamma_{p}-\right.$ $\left.\gamma_{\mathrm{m}}\right) \pi d^{2} / 2$ : thus, $\gamma_{\mathrm{p}}(r=0)=\gamma_{\mathrm{m}}$. consistent with the physical picture of a normal bilayer not interrupted by pores. The surface tension $\gamma_{n}$ of the pore wall is only defined for $r>0$, i.e., $N \geqslant 1$.

\subsection{Electric polarization terms}

The continuum expression for the electric polarization of material of volume $v$ in a homogeneous electric field $E$ is given by

$\boldsymbol{M} \boldsymbol{f}=\boldsymbol{c}_{0}(\boldsymbol{c}-1) \cdot \boldsymbol{E}$

where $\epsilon_{u}$ is the permittivity of a racuum and $\epsilon$ the relative dielectric permittivity. Since during electroporation bilayer volume disappears at the expense of solvent-filled pores, the $M$ values in eq. 9 are $M_{\mathrm{n}}>0$ and $M_{\mathrm{m}}<0$. At higher ionic strengths $(\geqslant 1 \mathrm{M})$ the electric conductivity of an aqueous solvent phase is so much larger than that of the planar bilayer that the applied voltage $V$ only drops across the bilayer (fig. 4). In terms of the constant field approximation, the average field in the bilayer is given by $E_{\mathrm{m}}=V / d$ and the field strength in the bulk electrolyte may be approximated by $E_{\mathrm{s}} \approx 0$.

The electric field within the solvent-filled pore is inhomogeneous. decreasing from the value $E_{m}$ in the pore wall/solvent interface toward the pore 
center. We now define a layer of solyent molecules adjacent to the inner cylindrical part of the pore wall. of thickness $\Delta r^{*}$ (fig. 1) within which the field intensity $E_{p}$ is approximated by $E_{p}=E_{m}$. For larger pores where $r>\Delta r^{*}$, the electric field strength in the region $r>\Delta r^{*}$ is considered to be $E_{\mathrm{s}} \approx 0$. For small pores where $r \leqslant \Delta r^{*}$, the homogeneous field approximation $E_{\mathrm{p}}=E_{\mathrm{m}}$ holds.

According to Jordan [23], at $1 \mathrm{M}$ ionic strength the electric field becomes highly inhomogeneous if $r \geqslant d / 5$. For these cunditions the relation $r=\Delta r^{*}$ $\approx d / 5$ specifies the largest pore size $N^{*}$ to which the small-pore field approximation $E_{\mathrm{p}}=E_{\mathrm{m}}$ may be applied. Using eq. 1 and $d=3.3 \mathrm{~nm} \mathrm{[12]} \mathrm{and}$ $I=0.9 \mathrm{~nm}$ we obtain $N^{*}=4.6$, as an average value for oxidized cholesterol; at $0.1 \mathrm{M}$ ionic strength (I), $N^{*}$ increases with decreasing $I$ [23].

\subsubsection{Smull pores}

Th: small-pore approximation is characterized by $r<\Delta r^{*}, E_{\mathrm{p}}=E_{m}, N \leqslant N^{*}$ and $E_{\mathrm{s}} \approx 0$. According to eq. 14, the electric polarization of the water-filled smali pore of volume $v_{p}=\pi r^{2} d$ is given by

$M_{\mathrm{p}}=\epsilon_{\mathrm{o}}\left(\epsilon_{w}-1\right) \pi r^{2} d E_{\mathrm{m}}$.

where $\epsilon_{w}$ is the relative dielectric permittivity of the electrolyte solution. The loss of polarization due to the decrease of the normal planar bilayer part during pore formation is given by

$M_{\mathrm{m}}=\epsilon_{\mathrm{o}}\left(\epsilon_{\mathrm{m}}-1\right)\left(-\pi r^{2} d\right) E_{\mathrm{mr}}$

Insertion of eqs. 15 and 16 into eq. 9 and integration yields

$\Delta \ddot{F}_{\mathrm{el}}={ }_{2}^{1} \epsilon_{\mathrm{o}}\left(\epsilon_{\mathrm{m}}-\epsilon_{\mathrm{w}}\right) \pi r^{2} d E_{\mathrm{m}}^{2}$

because $\epsilon_{\mathrm{w}}>\epsilon_{\mathrm{m},}, \Delta \bar{F}_{\mathrm{cl}}<0$, and the electric poiarization contribution favours pore formation. Introducing now the pore size $N$ from eq. 1 and using $E_{\mathrm{m}}=V / d$ we obtain

$\Delta \tilde{F}_{\mathrm{e}}=\beta(V) \frac{\epsilon_{\mathrm{m}}-\epsilon_{\mathrm{w}}}{\epsilon_{\mathrm{m}}-1} N^{2}$

with

$\beta(V)=\epsilon_{0}\left(\epsilon_{m}-1\right) I^{2} V^{2} /(8 \pi d)$

\subsubsection{Large pores}

In large pores $\left(r>\Delta r^{*}\right)$ it is only the solvent ring of volume $\pi\left[r^{2}-\left(r-\Delta r^{*}\right)^{2}\right] d$ that is appreciably polarized by $E_{\mathrm{m}}$. For the rest of the pore. $E=0$. Analogous to eqs. 15-17 we have

$\Delta \vec{F}_{e t}=-\epsilon_{0} \epsilon_{m}^{2} \pi d\left\{\left(\epsilon_{w}-1\right)\left[r^{2}-\left(r-\Delta r^{*}\right)^{2}\right]-\left(\epsilon_{m}-1\right) r^{2}\right\}$

and, after introducing $\Delta r^{*}=N^{*} l_{j}^{\prime}(2 \pi)$ and $r=$ $N I /(2 \pi)$ from eq. 1 and $E_{m}=V / d$, we obtain

$\Delta \vec{F}_{\mathrm{el}}=\beta(V) N^{2}+\mathrm{X}(V) N+C(V)$

where

$\lambda(V)=-\epsilon_{\mathrm{o}}\left(\epsilon_{w}-1\right) N^{*} l^{2} V^{2} /(4 \pi d)$

$C(V)=\epsilon_{0}\left(\epsilon_{w}-1\right)^{2} l^{2}\left(N^{*}\right)^{2} /(8 \pi d)$

and $\beta(V)$ is defined by eq. 19 .

\subsection{Free energy of electroporation}

Recalling eqs. 7, 12, 18 and 21 , the specific expressions for the characteristic Helmholtz free energy change of a single pore of size $N$ are:

(a) small pores $\left(r<\Delta r^{*}, N<N^{*}\right)$ :

$\Delta \tilde{F}(\mathrm{p})=\left[\beta(V) \frac{\epsilon_{\mathrm{m}}-\epsilon_{\mu}}{\epsilon_{\mathrm{m}}-1}+\beta_{0}\right] N^{2}+x_{0} N+C_{0}$

(b) large pores $\left(r>\Delta r^{*}, N>N^{*}\right)$ :

$\Delta \bar{F}(\mathrm{p})=\left[\beta(V)+\beta_{0}\right] N^{2}+\left[x(V)+x_{0}\right] N+C(V)+C_{0}(24)$

Eqs. 23 and 24 permit calculation of the energy profiles as a function of the pore size and transmembrane volta.e. For this purpose we use the numerical values if an experimental bilayer system: oxidized chc esterol [12,19]. The cata set $\gamma_{\mathrm{m}}=10^{-3} \mathrm{~N} / \mathrm{m}, \gamma_{\mathrm{p}}=1.9 \times 10^{-3} \mathrm{~N} / \mathrm{m}[19,24], d$ $=3.3 \mathrm{~nm}, \epsilon_{\mathrm{m}}=2.1[12,20] . l=0.9 \mathrm{~nm}, T=-313 \mathrm{~K}$. and $2 \leqslant N^{*} \leqslant 6$ was introduced into the expressions for $\beta, \chi$ and $C$ (eqs. 13, 19 and 22).

It is remarked that $\gamma_{\mathrm{p}}$ may be derived from $x_{0}$ by eq. 13. The edge energy $\left(\gamma^{*}=10^{-11} \mathrm{~N}[11,15]\right)$ of our model is given by $x_{0} / l=0.9 \times 10^{-11} \mathrm{~N}$ : with this value eq. 13 vields $\gamma_{p}=1.9 \times 10^{-3} \mathrm{~N} / \mathrm{m}$. consistent with previous estimates.

In fig. 5 the results of the computations are represented graphically in terms of the molar value $\Delta \tilde{F}=N_{\mathrm{A}} \Delta \tilde{F}(\mathrm{p})$, where $N_{\mathrm{A}}$ is the LoschmidtAvogadro constant, relative to the molar thermal energy $R T$ at various transmembrane voltages for 


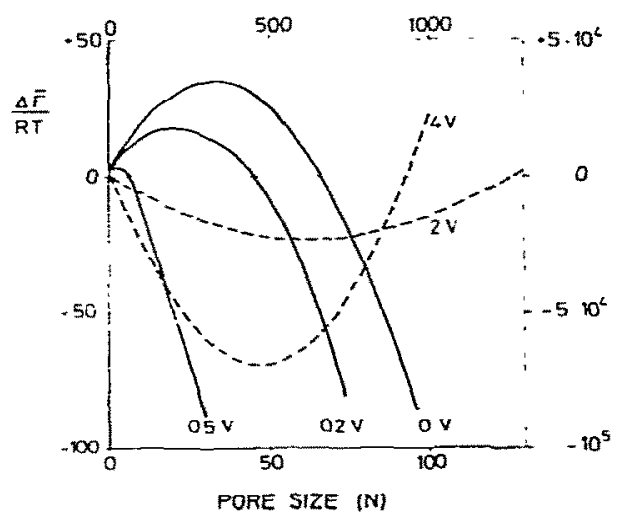

Fig. 5. Change in the characteristic Helmholy free energy i $\vec{F}$ relutute w the thermal energy $(R T)$ as a function of the pore sre. $\checkmark$ in planar bilayer at various transmembrane voltages.

$N^{*}=6$ (sec section 3.2 ). In accord with previous analysis of pore formation [11.14-16] there is a larger energy barrier for pore formation at low transmembrane voltage. At $\Lambda=0, \Delta \hat{F} / R T=C_{0}=$ 3.5. In previous models for small pores this ratio (60-80 [14]) is appreciably larger. Thus, in our model there is a finite chance for small pores: in accordance with experimental experiences the number of pores at $y=0$ is very small.

It is found that at a critical membrane voltage $I^{-(1)}=0.4 \mathrm{~V}$ the barrier for pore formation disappars and in the range $0.4 \mathrm{~V} \leqslant V \leqslant 1.15 \mathrm{~V}$ the pore size may increase unlimited at a constant voltage. The value of $V_{c}^{(1)}$ computed with the data set for oxidized cholesterol (without any parameter fitting) is in good agreement with the expermental value $(=0.4 \mathrm{~V}$. ref. 28 . fig. 11) of this system.

At higher voltage our model shows a qualitatively new phenomenon: there is a second critical voltage 19 . beyond which a global minimum ir. $\triangle F / R T$ appears at large pore sizes $(N \geqslant 500)$. l.arev pores at $V>V^{21}$ become stationary stable when the (unfavourable) surface tension terms are batanced by the (favourable) eleciric polarization term. (sec eys. 7 and 24 ). Eq. 24 sields the second critical voltage

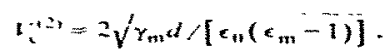

$y^{-12}=1.15 \mathrm{~V}$ for the data set of oxidized cholesierol.
The pore size of the stable single pores at $V>V_{c}^{(2)}$ in the supercritical voltage range depends on the actual vaiue of the transmembrane voltage; it approaches asymptotically a limit value

$N_{\mathrm{tmm}}=\lim _{V \rightarrow \infty}=\frac{-X(V)}{2 \beta(V)}=\frac{\varepsilon_{n}-1}{\epsilon_{m}-1} N^{*}$

With $\epsilon_{\mathrm{h}}=80, \epsilon_{\mathrm{m}}=2.1, N_{\mathrm{lim}}=72 N^{*}$. For $N^{*}=6$. $N_{\text {lim }}$ is much larger than any $N$ value corresponding to the barrier maximum $(N \approx 40)$ in fig. 5 . Therefore. when the voltage is switched off the pore is already so large that the size increases further until rupture (dielectric breakdown) occurs. However. in any real case of a Müller-Rudin hilayer there will be several pores developing in a planar bilayer. Pores of increasing size may overap and the membrane may rupture before the region of stable pore size for single pores is atlained.

\section{Thermodynamics of electroporation in vesicles}

Tiansient permeability changes caused by electric field pulses were first found in vesicular cell organelles [1]. Because of the lack of the buffering torus (of planar bilayer systems) electroporation in vesicles is associated with different surface changes. In addition, whereas planar bilayers are metastable systems the bilayers of large vesicles may be considered as equilibrium systems. The reference areas for the field-induced pore formation are the curved inner and outer bilayer surfaces of the area $A$, in the absence of pores. When pores are present the total surface of the vesicle exposed to solvent is larger than $A$, by the inner cylindrical area $2 \pi r d$ of a pore of radius $r$. The change in surface area $\Delta A$ during the formation of a single pore is given by

$J A=3 A_{\mathrm{p}}+\mathcal{A}_{\mathrm{m}}=A_{\mathrm{v}}+2 \pi r d-A_{\mathrm{V}}$

Analogous to the treatment of planar bilayers our periodic block model yields:

$\Delta A_{p}-2=\left\{r+\left[(r+d / 2)^{2}-r^{2}\right]\right\}$.
$\Delta A_{m}=-2=\left[(r+d / 2)^{2}-r^{2}\right]$

for pores in vesicles of radius $r_{3}$. Pore formation in 
larger vesicles $\left(r_{v} \gg r\right)$ of (slightly) curved bilayers may be treated in the same manner as planar bilayers. Therefore, we apply eq. 8 for the surface tension term:

$$
\begin{aligned}
\Delta \check{F}_{v_{, \gamma}} & =\gamma_{\mathrm{p}} \Delta A_{\mathrm{p}}+\gamma_{\mathrm{m} .} \Delta A_{\mathrm{m}} \\
& =\left(2 \gamma_{\mathrm{p}}-\gamma_{\mathrm{m}}\right) / \mathrm{d} N+\left(\gamma_{\mathrm{p}}-\gamma_{\mathrm{m}}\right) \pi d^{2} / 2 \\
& =\chi_{v} N+C_{\mathrm{o}}
\end{aligned}
$$

and eq. 9 for the electric polarization terms.

Finally, the changes in the characteristic Helmholtz free energy of the formation of a single pore in a vesicle are:

(a) small pores $\left(r \leqslant \Delta r^{*}\right)$

$\Delta \bar{F}_{v}(p)=\beta(V) \frac{\epsilon_{m}-\epsilon_{u}}{\epsilon_{m}-1} N^{2}+x_{0} N+C_{0}$

(b) large porzs $\left(r>\Delta r^{*}\right)$

$\Delta \tilde{F}_{\mathrm{v}}(\mathrm{p})=\beta(V) N^{2}+\left[x(V)+\chi_{0}\right] N+C(V)+C_{0}$

where $\beta, \chi$ and $C$ are defined by eqs. 13, 19 and 22.

In fig. 6 the stability regio: s of pores in vesicles are shown at various transmembrane voltages in terms of the molar ratio $\Delta \bar{F}_{\mathrm{v}} / R T$. In the framework of our model, pore formation in vesicles is always reversible and no rupture occurs. In reality, however, this feature only applies to large vesicles and not to alectric fields of long duration. Longlasting high fields may deform the shape of the vesicle $[25-27]$. Eqs. 30 and 31 and fig. 6 therefore

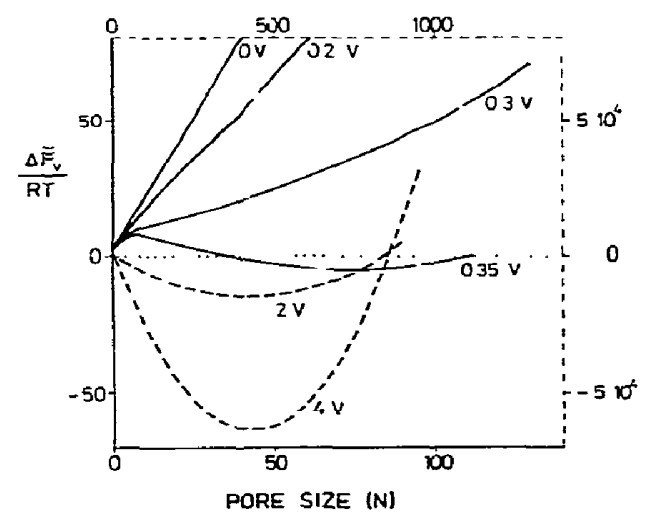

Fig. 6. The characteristic llelmholtz free energy change of pore formation in vesicular bilayer membranes. as a function of pore size at various transmembrane voltages, $V$; see fig. 5 . only apply to short $(<1-10 \mu \mathrm{s})$ pulses. The results presented in fig. 6 are obtained usirg the same values as those tised for the planar bilayer system of oxidized cholesterol (section 3.3). However, for vericles the critical voltage $V_{c}^{(1)}=V_{c}^{(2)}=V_{c}$ (corrcsponding to $\left[\partial\left(\Delta \bar{F}_{v} / R T\right) / \partial N\right]=0$ at $\left.N=N^{*}\right)$ is $0.32 \mathrm{~V}$; it is smaller than that $(0.4 \mathrm{~V})$ of the planar bilayar system. The equilibrium pore size decreases with increasing transmembrane voltage until apparently a limit size is attained.

\section{Dynamics of electroporation}

Pore iormation and resealing of ipid bilayers may be treated as a stochastic process in terms of a Markov chain. The fundamental pore states (a) and the state transitions between nearest neighbours are shown in fig. 3 . The general stochastic state transitions are given by the scheme

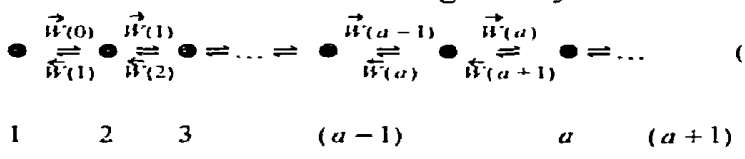

where $\vec{W}(a)$ and $\vec{W}(a)$ represent the 'rate constants' or transition probabilities per unit time for the steps $a \rightarrow(a+1)$ and $a \rightarrow(a-1)$, respectively.

\subsection{Transition probabilities}

Denoting by $\Delta^{\cdot}$ a small time interval within which one state transition $a \rightarrow(a+1)$ occurs, the transition probability of this state change is given by

$$
\vec{W}(a) \Delta t=\left(4 a^{2} \lambda t / \tau\right) \exp \left(-\lambda \vec{F}_{N . v+1} / k T\right)
$$

where $\tau$ is the characteristic transition time and $1 / \tau$ the transition frequency representing the number of trials per unit time; note that $N=2 a$ (fig. 3 ). The lactor $4 a^{2}=N^{2}$ describes the number of possible $a \rightarrow(a+1)$ transitions. The rate-limiting step of this transition is the diffusion of lipids from tha bulk bilayer into the pore wall; that may occur at $N$ different sites in one layer of the pore wall or at $N^{2}$ sites independently in both layers of the bilayer (fig. 1). $\Delta \bar{F}_{N, N+1}$ refers to the free energy difference between pore size $N+1$ and $N$. Note that the surface energy of the periodic block 
structure in a pore wall is lower by the energy $\alpha$ compared to that of the aperiodic structure (fig. 3).

We now apply eqs. 23 and 24 to three limiting cases:

(a) $N+1<N^{*}$ (small pores) associated with the transition probability $W(a)=\ddot{w}^{\prime}(a)$,

$$
\begin{aligned}
د \bar{F}_{N \ldots+1}= & {\left[\beta(V) \frac{\epsilon_{u}-\epsilon_{m}}{\epsilon_{\mathrm{m}}-1}+\beta_{0}\right](N+1)^{2}+x_{0} N+C_{0}+\alpha } \\
& -\left[\beta(V) \frac{\epsilon_{m}-\epsilon_{w}}{\epsilon_{m}-1}+\beta_{0}\right] N^{2}+x_{0} N+C_{0} \\
= & (4 a+1)\left[\beta(\nu) \frac{\epsilon_{m}-\epsilon_{w}}{\epsilon_{m}-1}+\beta_{0}\right]+\alpha .
\end{aligned}
$$

(b) $N \leqslant N^{*} \leqslant N+1, \dot{W}(a)=\dot{w}^{\prime \prime}(a)$ :

$$
\begin{aligned}
& د \bar{F}^{\prime \prime}, v+1=\left[(2 a+1)^{2}-4 a^{2} \frac{\epsilon_{m} \cdots \epsilon_{u}}{\epsilon_{m}-1}\right] \beta(V) \\
& +(4 a+1) \beta_{0}+x(V)+C(V)+\alpha
\end{aligned}
$$

(c) $N>N^{*}$ (large pores) associated with $\dot{W}(a)=$ ii (a):

$د \tilde{F}_{1, \because+1}=(4 a+1)\left[\beta(V)+\beta_{0}\right]+\chi(V)+\alpha$

The state transitiori $a \rightarrow(a-1)$ is dominant during the resealing of pores. Analogous to tq. 33 the transition probability of the $a \rightarrow(a-1)$ step is given by

$\bar{b}(a) \Delta r=\left(a^{2} s_{t} / \tau\right) \exp \left(-\Delta \bar{F}_{N, v-1} / k T\right)$

The rate-limiting step of the reseaiing process is the movement of a non-rotated block (figs. 2 and 3) from the pore wall edge into the bulk bilayer. Within one layer annealing of a non-rotated block can be realized $N / 2$ times and in the two layers the number of realizations is $(N / 2)^{2}$. Analogous to eqs. 34-36 we obtain:

(a) $N<N^{*}$ (small pores), $\dot{W}(a)=\bar{w}^{\prime}(a)$,

$د \bar{F}_{i,-1}=(1-4 a)\left[\beta(V) \frac{\epsilon_{m}-\epsilon_{w}}{\epsilon_{m}-1}+\beta_{0}\right]-2 x_{0}+\alpha$

(b) $(N-1) \leqslant N^{*} \leqslant N ; \dot{W}(a)=\ddot{u}^{\prime \prime}(a)$

$\Delta \tilde{F}: \checkmark-1=\left[(2 a-1)^{2} \frac{\epsilon_{\mathrm{m}}-\epsilon_{\mathrm{u}}}{\epsilon_{\mathrm{m}}-1}-4 a^{2}\right] \beta(\nu)$

$$
+(1-4 a) \beta_{0}-2\left[x_{0}+a x(V)\right]-C(V)+\alpha
$$

(c) $(N-1)>N^{*}$ (large pores), $\bar{W}(a)=\bar{u}(a)$.

$\Delta \bar{F}_{\mathrm{s} . .+1}=(1-4 a)\left[\beta(V)+\beta_{0}\right]-x(V)-2 x_{0}+\alpha$

For the states $a=0$ and $a=-1$, the transition probabilities are

$\vec{W}(0)=\tau^{-1} \exp \left(-\Delta \tilde{F}_{0.1} / k T\right)$

$\vec{W}(-1)=0$.

\subsection{Master equation of eleciroporation}

The probability of occurrence of state $a$ at time $t+\Delta t$ is given by

$$
\begin{aligned}
P(a \cdot t+\Delta t)= & \vec{W}(a-1) \Delta t P(a-1, t) \\
& +\bar{W}(a+i) \Delta t P(a+1, t) \\
& +\{1-[\ddot{W}(a) \Delta t+\bar{W}(a) \Delta t]\} P(a, t)
\end{aligned}
$$

The difference equation (eq. 42) may be transformed into the master equation

$$
\begin{aligned}
\frac{\mathrm{d} P(a, t)}{\mathrm{d} t}= & \bar{W}(a-1) P(a-1 . t)+\bar{W}(a+1) P(a+1, t) \\
& -[\vec{W}(a)+\bar{W}(a)] P(a, t)
\end{aligned}
$$

Experimental data refer to average values of pore size and to the variance of fluctuations in the pore size. The average pore state $a$ is related to the average pore size $\langle N\rangle$ by $\langle a\rangle=\langle N\rangle / 2$. From eq. 43 we readily obtain

$$
\frac{\mathrm{d}\langle a\rangle}{\mathrm{d} r}=\frac{\mathrm{d}}{\mathrm{d} t} \sum_{a=0}^{\infty} a P(a, t)=\sum_{a=0}^{\infty}[\vec{w}(a)-\bar{W}(a)] P(a \cdot t)
$$

Assuming now a Poisson process, eq. 44 is transformed into a non-linear ordinary differential equaiion (see the appendix):

$$
\begin{aligned}
& \frac{\mathrm{d}\langle a\rangle}{\mathrm{d} t}=\sum_{a=0}^{\mathrm{ENT} N^{*} / 2}\left\{\left[\vec{w}^{\prime}(a)-\bar{w}^{\prime}(a)\right]-[\vec{w}(a)-\bar{w}(a)]\right\} P(a, t) \\
& \quad+\left\{\left[\overrightarrow{w^{\prime}}\left(a^{\prime}\right)-\bar{w}^{\prime \prime \prime}\left(a^{\prime}\right)\right]-\left[\overline{w^{\prime}}\left(a^{\prime}\right)-\overline{w^{\prime}}\left(a^{\prime}\right)\right]\right\} P\left(a^{\prime} \cdot t\right)+\langle\vec{w}-\bar{w}\rangle
\end{aligned}
$$

where the definition $a^{\prime}=\operatorname{ENT}\left(N^{*} / 2+1\right)$ holds and ENT is the integer of a numerical value $p$ qrs; e.g. $\operatorname{ENT}(p$. qrs $)=p$.

It is remarked that the pore formation corresponds to $\mathrm{d}\langle a\rangle / \mathrm{d} t>0$ and that the resealing of the membrane refers to $\mathrm{d}\langle a\rangle / \mathrm{d} t<0$, formally.

\subsection{Stable and unstable stationary solutions}

The stationary solutions $(\mathrm{d}\langle a\rangle / \mathrm{d} t=0)$ of the master equation (eq. 45) are repesented as a phase 


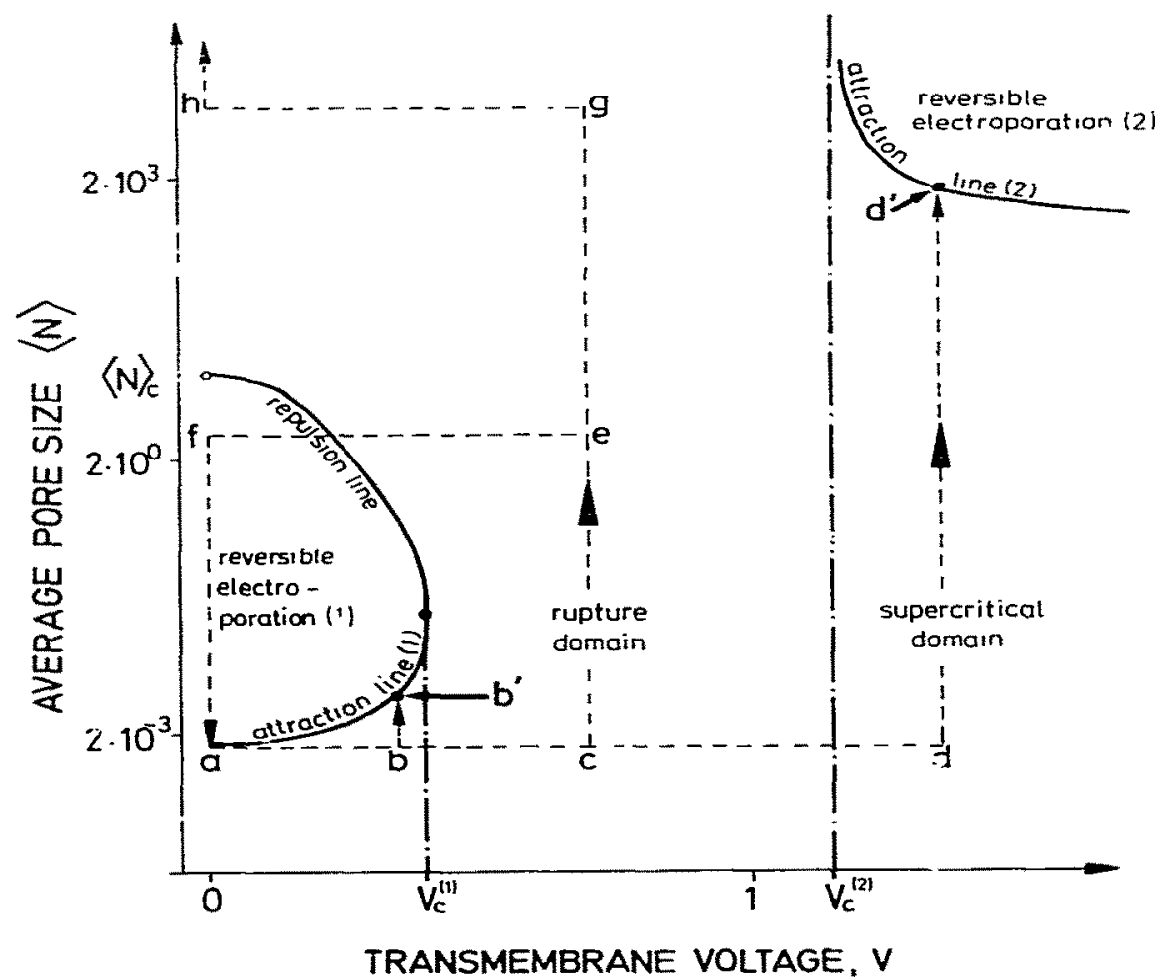

Fig. 7. Phase diagram of electroporation in lipid bilayers. The solid lines represent the stationary solutions of the electroporation master equation (eq. 45) of the text: the lines separate two reversible electroporation domains from regions of nonstationary states where either resealing of the pore $\left(\langle N\rangle\left\langle\langle N\rangle_{c}\right.\right.$ ) or rupture (dielectric breakdown) of the bilayer may occur ( $\left.\langle N\rangle\right\rangle\langle N\rangle_{\varepsilon}$ ). See text.

diagram in fig. 7. There are two lines in this diagram (attraction lines) on which the average pore size is stationary and stable at a given transmembrane voltage. The phase diagram contains two domains which are associated with critical voltages $V_{c}^{(1)}$ and $V_{c}^{(2)}$, respectively; $V_{c}^{(2)}>V_{c}^{(1)}$. They are called reversible electroporation domains ( 1 and 2). Within these domains the right-hand side of eq. 45 is negative, i.e., resealing to a smaller stationary pore size occurs. Outside these reversible electroporation domains the right-hand side of eq. 45 is positive, i.e., the pore size increases at a given voltage.

For $V=0$ and $N^{*}=6$ our model yields the stable stationary solution, $\langle N\rangle_{0}=1.5 \times 10^{-3}$, i.e., the average value of $N$ in the absence of a transmembrane voltage is very small. At $V \leqslant V_{c}^{(1)}$ the pore size $\langle N\rangle$ increases but remains small: switching off the voltage in this range leads to a (reversible) return of the system to $\langle N\rangle_{0}$ (see, for in stance, the pathway $a \rightarrow b \rightarrow b^{\prime} \rightarrow a$; fig. 7).

The two reversible electroporation domains are separated from each other by a rupture domain. Within the transmembrane voltage range $V_{c}^{\text {(1) }}<V$ $\left\langle V_{c}^{(2)}\right.$ the pore size at constant voltage may increase unlimited until the membrane ruptures. If, however, the voltage is switched off before the pore size has attained a critical value $\langle N\rangle_{c}$, the system 'jumps' into the reversible electroporation domain (1) and is 'attracted' to the stable stationary attraction line (1) (see, for example, the pathway $a \rightarrow c \rightarrow e \rightarrow f \rightarrow a$; fig. 7). When the pulse lasts longer such that $\langle N\rangle\rangle\langle N\rangle_{c}$, switching off the voltage, for instance, at point $g$ causes $\langle N\rangle$ to 
trace the pathway $g \rightarrow h$; at $h$ the system is further 'repelled' toward higher $N$ values until rupture cucurs.

The transmembrane voltage range $V>V_{i}^{(2)} \mathrm{de}-$ fines a supercritical domain and the reversible electroporation domain (2). If an electric pulse of $y>V^{-2}$ lasts long enough, a 'second attraction line of stable stationary pore sizes is attained: for instance, along the pathway $a \rightarrow d \rightarrow d^{\prime}$. At higher $V$ values the average pore size on the attraction line (2) decreases slightly toward a saturation value. Switching off the voltage at point $d^{\prime}$ leads to membrane rupture because $\left.\left\langle N\left(d^{\prime}\right)\right\rangle\right\rangle\langle N\rangle_{c}$.

It is emphasized that the phase diagram in fig. 7 refers to the average pore size. However. there may be large fluctuations in $N$. At $V<V^{(1)}$, for example, at point $b$ ' the 'distance' $\Delta\langle N\rangle$ between point $b^{\prime}$ on the attraction line (1) and the corresponding point on the repulsion line at $V=V\left(h^{\prime}\right)$ is comparable to the fluctuations in $N$. Therefore, after the first passage time the pore size can exceed the unstable stationary value ors the repulsion line and can increase unlimited unil the membrane ruptures.

Within the typical range $\left(2<N^{*}<6\right)$ of the small-pore approximation the form of the phase diagram is oniy slightly cependent on $N^{*}$ (not shown in fig. 7).

\section{Kinetics of electroporation}

\subsection{Pore formation}

The master equation for the average pore size, eq. 45 . may be numerically integrated in the form

$(\bar{J} / \tau) \exp (-\alpha / k T)=\int_{\langle a(0)\rangle}^{(a(r)\rangle} \frac{\mathrm{d}\langle a\rangle}{f(\langle a\rangle, V)}$

where $f(\langle a\rangle . V)$ is the right-hand side of eq. 45 . $\langle a(0)\rangle$ the stable stationa:y value at $V=0$ and $\langle a(t)\rangle$ corresponds to the average pore size at time $t$ after application of $V$ at $t=0$. Eq. 46 permits the calculation of the average time interval $\overline{J t}$ within which a given pore size is obtained. For instance. $\overline{\Delta t}$ may refer to the time interval where the pore starts to conduct hydrated alkali metal ions.

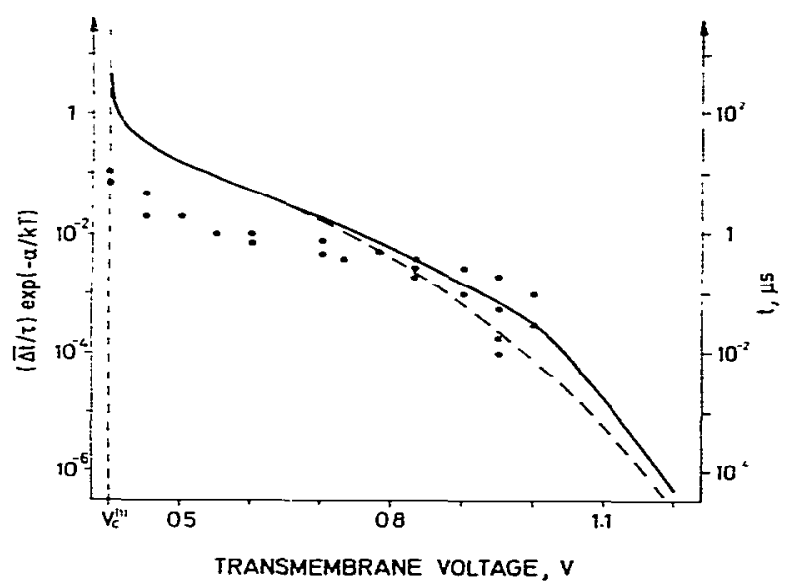

Fig. 8. The interval $\bar{J}$ of building up of the lower limit pore size $\left\langle N_{\mathrm{sh}}\right\rangle$ for the conduction of alkali metal ions. normalized by the sealling factor $\tau^{-1} \exp (-\alpha / k T)$. as a function of the transmembrane voltage, $V$. at threshold pore size $\left\langle N_{\mathrm{th}}\right\rangle=2$ $(-\ldots--)$ and at $\left\langle N_{\mathrm{th}}\right\rangle=6(-)$. If the scaling factor is $10^{4} \mathrm{~s}^{-1}$ the ordinate on the right-hand side marks the absolute time scale of $\overline{i t}$. (ब) Experimental data of Benz and Zimmermann [28].

It is found that $\overline{\Delta t}$ is almost invariant to $N^{*}$ within $2 \leqslant N^{*} \leqslant 6$. However, the smaller the transmembrane voltage the larger is $\overline{\Delta t}$; at $V=V_{c}^{(1)}$. $\overline{\Delta t} \rightarrow \infty$. Fig. 8 is a graphical representation of eq. 46 for two values of $N^{*}$. Experimental data of Benz and Zimmermann (ref. 28: fig. 11) can be satisfactorily fitted when the scaling factor $\left(\tau^{-1} \exp [-\alpha / k T]\right)$ is $10^{4} \mathrm{~s}^{-1}$. The deviations of the calculated curves from the experimental data at longer voltage durauons (charging times) may arise from the actual fluctuations in the pore size, which increase with increasing pulse durations. In particular, if $V \approx V_{c}^{(1)}$, fluctuations in $N$ may lead to permeability changes and concomitant ion conductance which are much larger than those predicted for the average value $\langle N\rangle$ of the pore size.

\subsection{Resealing of ion conducting bilayers}

When the electric pulse is switched off before the critical pore size $\langle N\rangle_{\mathrm{c}}$ is attained, the membrane system (at $V=0$ ) is in the reversible electroporation domain (1) and reseals to the stationary pore size $\langle N\rangle_{0}$. In order to compare with experimental data on resealing times of oxidized 


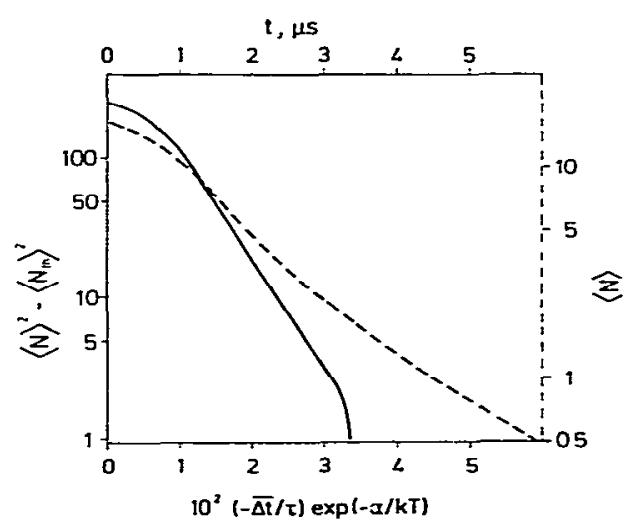

ᄃig. 9. Relative time cuurse of the pore resealing process in terms of the average poor size $\langle N\rangle(----)$ according to eq46. Resealing is considared as the reverse of pore formation formally replacing $\overline{\Delta_{r}}$ by $\left(-\overline{\Delta_{t}}\right.$ ) (see fig. 8). The solid line represents the transfo:mation from $\langle N\rangle$ to $\langle N\rangle^{2}-\left\langle N_{\mathrm{th}}\right\rangle^{2}$. with $\left\langle N_{\mathrm{th}}\right\rangle=1.5$ [20]. If the same scaling factor $10^{3} \mathrm{~s}^{-1}$ (as for pore formation) is used for the resealing process, the upper abscissa marks the absolute time scale for $\langle N(t)\rangle$.

cholesterol [20], we may formally treat the resealing process as the reverse of the pore formation. For the calculation of resealing times we replace $(\overline{\Delta t})$ in eq. 46 by the term $(-\overline{\Delta t})$, where $(-\overline{\Delta t})$ may represent the time interval of resealing after switching off the voltage. The resealing process is initially not a simple exponential when $\langle N\rangle$ is far from the stable stationary value.

In fig. 9 the dashed curve represents eq. 46 in terms of the relative resealing time given by $[(-\overline{\Delta t}) / \tau] \exp (-\alpha / k T)$. The solid curve is calculated from eq. 46 in terms of $\langle N\rangle^{2}-\left\langle N_{\mathrm{th}}\right\rangle^{2}$ where $\left\langle N_{\mathrm{th}}\right\rangle$ is the average threshold pore size [20]. For $\langle N\rangle\left\langle\left\langle N_{\mathrm{th}}\right\rangle\right.$ no ions are conducted anymore. The shape of this (solid) curve is the same as that of the experimental conductivity vs. time curves [20]. Therefore, as is physically plausible, the ion conductance is proportional to $N^{2}$ (i.e., the surface of the pore mouth).

When we now use the same scaling factor $\tau^{-1} \exp (-\alpha / k T)=10^{4} \mathrm{~s}^{-1}$ as for the pore formation, the time constant associated with the linear section of the function $\ln \left(\langle N\rangle^{2}-\left\langle N_{\mathrm{th}}\right\rangle^{2}\right)$ is found to be $0.55 \mu \mathrm{s}$. This value is exactly the relaxation time of the faster part of the experimental resealing process at $40^{\circ} \mathrm{C}[20 \mathrm{j}$.
In summary, our periodic block model for pores in lipid bilayers appears to be consistent with basic features of observed electroporation phenomena. The fundamental process of pore formation is the block rotation by about $90^{\circ}$ comprising (two) nearest-neighbour lipids. The driving force for block rotation in electric fields is the electric polarization of the polar solvent molecules (adjacent to the polar head groups of the lipids) which are transported by lipid rotation into the region of the larger electric field spreading irom the pore wall into the solution of the pore interior.

\section{Appendix}

\section{A1. Master equation for $\langle a\rangle$}

Substitution of the respective specific values of $\vec{W}(a)$ and $\bar{W}(a)$ into eq. 44 finally yields:

(a) for $a^{\prime}<\left(N^{*} / 2\right)<\left(a^{\prime}+0.5\right)$,

$$
\begin{aligned}
& \frac{\mathrm{d}\langle a\rangle}{\mathrm{d} t}=\sum_{a=0}^{[E N T(N) / 2\rangle]-\mathrm{I}}\left[\overline{n^{\prime}}(a)-\overline{n^{\prime}}(a)\right] P(a, t) \\
& +\left\{\left[\bar{w}^{\prime \prime}\left(a^{\prime}\right)-\bar{w}^{\prime}\left(a^{\prime}\right)\right] P\left(a^{\prime}, t\right)\right\} \\
& +\sum_{a-\left\{\operatorname{ENT}\left(N^{-} / 2\right)\right\}+1}^{\infty}[\vec{w}(a)-\bar{w}(a)] P(a . t) \\
& \text { [ENT } \left.\left(N^{*} / 2\right)\right]-1 \\
& =\sum_{a=0}^{[E N T(N-/ 2)]-1}\left\{\left[\vec{w}^{\prime}(a)-\vec{w}^{\prime}(a)\right]\right. \\
& -[\bar{w}(a)-\bar{w}(a)]\} P(a, t) \\
& +\langle\vec{w}-\bar{w}\rangle+\left\{\left[\vec{w}^{\prime \prime}\left(a^{\prime}\right)-\bar{w}^{\prime}\left(a^{\prime}\right)\right]\right. \\
& \left.-\left[\vec{w}\left(a^{\prime}\right)-\bar{w}\left(a^{\prime}\right)\right]\right\} P\left(a^{\prime}, t\right) .
\end{aligned}
$$

where $a^{\prime}=\operatorname{ENT}\left(N^{*} / 2\right)$ and $\langle\vec{w}-\bar{w}\rangle$ $=\sum_{a=0}^{\infty}[\bar{w}(a)-\bar{w}(a)] \cdot P(a, t)$,

(b) for $\left(a^{\prime}-0.5\right)<\left(N^{*} / 2\right)<a^{\prime}$.

$$
\begin{aligned}
\frac{\mathrm{d}\langle a\rangle}{\mathrm{d} t}= & \sum_{a=0}^{\mathrm{ENT}(N-/ 2)}\left\{\left[\vec{w}^{\prime}(a)-\bar{w}^{\prime}(a)\right]\right. \\
& -[\vec{w}(a)-\bar{w}(a)]\} P(a, t) \\
& +\left\{\left[\vec{w}\left(a^{\prime}\right)-\bar{w}^{\prime \prime}\left(a^{\prime}\right)\right]-\left[\vec{w}\left(a^{\prime}\right)-\bar{w}\left(a^{\prime}\right)\right]\right\} P\left(a^{\prime}, t\right) \\
& +\langle\bar{w}-\bar{w}\rangle
\end{aligned}
$$

where $a^{\prime}=\left[\operatorname{ENT}\left(N^{*} / 2\right)\right]+1$.

According to eqs. 33-41 the terms $\vec{w}(a)$ and 
iv.(a) may be expressed as

$\ddot{n}(a)=A a^{2} \exp (\omega a)$

$n(a)=B a^{2} \exp (-\omega a)$

with

$\omega \equiv 4\left[\beta(V)+\beta_{0}\right] / k T$

$A=\tau^{-} \operatorname{cxp}\left\{-\left[\beta(V)+\beta_{0}-\chi(V)-2 \chi_{0}+\alpha\right] / k T\right\}$

$B=(4 / \tau) \exp \left\{-\left[\beta(V)+\beta_{0}-\chi(V)-2 x_{0}+\alpha\right] / k T\right\}$

Because the values of $A$ and $B$ are independent of $a$ it is sufficient to determine the average:

$\left\langle a^{2} \exp ( \pm \omega a)\right\rangle=\sum_{a=0}^{\infty} a^{2} \exp ( \pm \omega a) \cdot P(a, t)$

We now define a generating function:

$F(s \pm \omega . t)=\sum_{a=0}^{\infty} \exp [(s \pm \omega) a] P(a, t)$

and a cumulant generating function

$$
\begin{aligned}
K(s \pm \omega . t)= & \ln F(s \pm \omega . t) \\
= & K(0 . t)+K^{\prime \prime}(0 . t)(s \pm \omega) \\
& +K^{\prime \prime}(0 . t) \frac{(s \pm \omega)^{2}}{2 !}+\ldots
\end{aligned}
$$

where $K(s \pm \omega, t)$ is expanded into a Taylor series around $s+\omega=0$.

Assuming that the electroporation is a Poisson process we have:

$P(a, t)=\langle a(t)\rangle^{a}[\exp (-\langle a\rangle)] / a !$

The cumulants are

$K^{\prime}(0,1)=0, K^{\prime}=K^{\prime \prime}=K^{\cdots}=\ldots=\langle a(1)\rangle$.

Substituting eq. A11 inis eq. A9 yields

$\kappa(s \pm \omega, t)=\langle a\rangle \sum_{i=1}^{\infty} \frac{(s \pm \omega)^{\prime}}{i !}=\langle a\rangle(\exp [s \pm \omega]-1)$

Consequently, the generating function is

$F(s \pm \omega, 1)=\exp [\langle a\rangle(\exp [s \pm \omega]-1)$.

Finally, $w$ and $i$, (eq. A3) are obtained from

$\langle u\rangle-\left|\frac{\mathrm{d}^{2} F}{\mathrm{~d} \omega^{2}}\right|_{,-0}=\sum_{a=0}^{\infty}\left[a^{2} \exp ( \pm \omega a)\right] P(a, t)$
Since

$\langle w\rangle-\langle a\rangle \exp ( \pm \omega)\left\{\exp \left[\langle a\rangle\left(e^{ \pm \omega}-1\right)\right]\right\}\left(1+\langle a\rangle e^{ \pm \omega}\right)$.

the final result is

$$
\begin{aligned}
\left\langle w^{w}-\dot{w}\right\rangle= & B\langle a\rangle e^{-\omega}\left\{\exp \left[\langle a\rangle\left(e^{-\omega}-1\right)\right]\right\}\left(1+\langle a\rangle e^{-w}\right) \\
& -A\langle a\rangle e^{\omega}\left\{\exp \left[\langle a\rangle\left(e^{\omega}-1\right)\right]\right\}\left(1+\langle a\rangle e^{\omega}\right) .
\end{aligned}
$$

\section{Acknowledgements}

We thank the Deutsche Forschungsgemeinschaft and the Hungarian Academy of Science for financial support.

\section{References}

1 E. Neumann and K. Rosenheck, J. Membrane Biol. 10 (1972) 279 .

2 U. Zimmerman, J. Schulz and G. Pilwat. Biophys. J. 13 (1973) 1005.

3 E. Neumann. G. Gerisch and K. Opatz. Naturwissenschaften 67 (1980) 414 .

4 P. Scheurich and U. Zimmermann, Z. Naturforsch. 35c (1980) 1081 .

5 H. Weber, W. Förster. H.E. Jacob and H. Berg. Z. Allg. Mikrobiol. 21 (1981) 555.

6 U. Zimmermann, F. Riemann ard G. Pilwat, Biochim. Biophys. Acta 436 (1976) 460.

7 U. Zimmermann. Biochim. Biophys. Acta 674 (1982) 227.

8 E. Neumann, M. Schaefer-Ridder, Y. Wang and P.H. Hofschneider, EMBO J. I (1982) 841.

9 J.M. Crowley. Biophys. J. 13 (1973) 711.

10 U. Zimmermann. G. Pilwat and F. Riemann. Biophys. J. 14 (1974) 885

11 J.Y. Abidor, V.B. Arakelyan, L.V. Chernomordik. Y.A. Chizmadzhev, V.F. Pastushenko and U.R. Tarasevich, Bioelectrochem. Bioenerg. 6 (1979) 37.

12 R. Benz. F. Beckers and U. Zimmermann, J. Membrane Bicl. 48 (1979) 181

13 J. Teissic and T.Y. Tsong. Biochemistry 20 (1981) 1548.

14 I.P. Sugár, J. Physiol. (Paris) 77 (1981) 1035.

15 J.D. Litster. Phys. Lett. 53a (1975) 193.

16 J.C. Weaver and R.A. Mintzer. Phys. Lett. 863 (1981) 57.

17 G.S. Hartley. Aqueous solutions of paraffinic chain salts (Hermann, Paris, 1936).

18 P. Fromherz, Chem. Phys. Lett. 77 (1981) 460.

19 H.T. Tien, Bilayer lipid membranes, theory and practise (Marcel Dekker. New York. 1974) p. 40.

20 R. Benz and U. Zimmermann. Biochim. Biophys. Acta 640 (1981) 169 . 
21 E.A. Guggenheim, Thermodynamics (North-Holland, Amsterdam, 1967) p. 47, 335.

22 E. Neumann, Stud. Biophys. 94 (1983) 107.

23 P.C. Jordan. Biophys. J. 39 (1982) 157.

24 V.F. Pastushenko, Y.A. Chizmadzhev and V.B. Arakelyan. Bioelectrochem. Bioenerg. 6 (1979) 53.

25 A.A. Füredi and I. Ohad. Biochim. Biophys. Acta 79 (1964) 1.
26 F. Pliquett, Z. Biol. 116 (1968) 10.

27 A.W. Friend, Jr, E.D. Finck and H.P. Schwan. Science 187 (1975) 357.

28 R. Benz and U. Zimmermann, Bioelectrochem. Bioenerg. 7 (1980) 723 . 